Bull. Korean Math. Soc. 51 (2014), No. 5, pp. 1339-1345

http://dx.doi.org/10.4134/BKMS.2014.51.5.1339

\title{
A BOUNDED KOHN NIRENBERG DOMAIN
}

\author{
Simone Calamai
}

\begin{abstract}
Building on the famous domain of Kohn and Nirenberg we give an example of a domain which shares the important features of the Kohn Nirenberg domain, but which can also be shown to be $\phi$-bounded. As an application, we remark that this example has compact automorphism group.
\end{abstract}

\section{Introduction}

Consider a domain $A \subset \mathbb{C}^{n}$ of smooth boundary. At a boundary point $p \in \partial A$ which is strongly Levi-convex, we can find a local system of holomorphic co-ordinates such that $\partial A$ is $\mathbb{R}$-convex around $p$; in short we can say that strongly Levi-convex points provide local $\mathbb{R}$-convexifiability. This is known as the Narasimhan lemma (see [9]). A long standing conjecture would extend the local $\mathbb{R}$-convexifiability to boundary points which are only weakly Levi-convex. But in 1972 J. J. Kohn and L. A. Nirenberg [6] found a counterexample to the conjecture. Their domain $D_{K N}$ is given by

$$
D_{K N}:=\left\{\left.(z, w) \in \mathbb{C}^{2}|\operatorname{Re} w+| z w\right|^{2}+|z|^{8}+\frac{15}{7}|z|^{2} \operatorname{Re} z^{6}<0\right\} .
$$

The domain is regular and real-analytic; the origin is a weakly Levi-convex boundary point, and all the other boundary points are strongly Levi-convex; the domain is not locally $\mathbb{R}$-convexifiable at the origin.

Until recently, a great deal of work was done, among the others, by M. Kolar (see [7], [8]), by J. Byun (see [2], [3]), and by J. Byun and H. R. Cho (see [4]) in order to understand the properties and peculiarities of convexifiability and of the domain $D_{K N}$.

Also, we feel that there is still a need of new examples which indicate in which directions theories can develop about the phenomenon of convexifiability.

To the author's knowledge, it is still not known whether there is a global biholomorphism of $D_{K N}$ whose image is a smoothly bounded domain of $\mathbb{C}^{2}$. In Section 2 we give a precise definition of the latter property and we refer to it as

Received May 23, 2012; Revised March 6, 2013.

2010 Mathematics Subject Classification. 32T15, 32T27.

Key words and phrases. Kohn Nirenberg domain, holomorphic boundedness.

The author is partially supported by GNSAGA. 
$\phi$-boundedness. We conjecture that the Kohn Nirenberg domain is $\phi$-bounded, and we can look at the result of these notes as a first step in that direction.

With this motivation, we state the result of this note.

Theorem 1.1. Consider the domain $D:=\left\{(z, w) \in \mathbb{C}^{2} \mid \rho(z, w)<0\right\}$, where the defining function $\rho: \mathbb{C}^{2} \rightarrow \mathbb{R}$ is given by

$$
\rho(z, w):=\operatorname{Re} w+\frac{1}{5}|w|^{2}+|z w|^{2}+|z|^{8}+\frac{15}{7}|z|^{2} \operatorname{Re} z^{6}+10|z|^{10}<0 .
$$

The domain $D$ is regular, bounded and real-analytic. The origin is a weakly Levi-convex boundary point, while all the others boundary points are strongly Levi-convex. Finally, the domain $D$ is not locally $\mathbb{R}$-convexifiable at the origin.

In short, we see that $D$, which is a slight modification of the Kohn Nirenberg domain, keeps the above listed main features of $D_{K N}$, and plus $D$ is bounded.

As an immediate application of Theorem 1.1, which makes use of a result by E. Bedford and S. Pinchuk [1], we have:

Corollary 1.2. The automorphism group of the domain $D$ is compact.

We would like to remark that also the original Kohn Nirenberg domain has compact automorphism group. Moreover, in [4], Byun and Cho were able to explicitly compute the automorphism group. We will observe that the automorphism group of the Kohn Nirenberg domain is contained in our modification. It would be interesting to study whether the methods used in [4] can be generalized to our domain.

The structure of this note is as follows. In Section 2, we make precise the definition of boundedness and of compactness of the group of holomorphic automorphisms. In Section 3 we present the proof of our theorem divided in lemmas which prove the different properties separately; then we discuss the corollary of the main result.

Acknowledgments. Thanks to Mario Landucci for bringing author's attention to the Kohn Nirenberg domain; thanks also to Daniele Angella for helpful discussions. Finally, thanks to the anonymous referee for valuable remarks.

\section{2. $\phi$-boundedness and the group of holomorphic automorphisms}

Let $A \subseteq \mathbb{C}^{n}$ be a domain of $\mathbb{C}^{n}$. We denote by $\operatorname{BiHol}\left(D, \mathbb{C}^{2}\right)$ the space of biholomorphic maps from $D$ to and open set of $\mathbb{C}^{2}$. From now on, we will also keep the Greek letter $\phi$ to denote an element of $\operatorname{BiHol}\left(D, \mathbb{C}^{n}\right)$.

Definition 2.1. We say that a domain $A \subseteq \mathbb{C}^{n}$ is $\phi$-bounded when there exists an element $\phi \in \operatorname{BiHol}\left(D, \mathbb{C}^{n}\right)$ such that the image $\phi(A)$ is bounded in the standard topology of $\mathbb{C}^{n}$.

In particular, if the given domain $A$ is already bounded in $\mathbb{C}^{n}$, we say that it is Id-bounded meaning that the domain is bounded when in particular $\phi$ is the identity map. 
Given a domain $A \subseteq \mathbb{C}^{n}$ of $\mathbb{C}^{n}$, we denote by $\operatorname{Aut}(A)$ the group of holomorphic automorphisms of $A$, that is the maps $\psi \in \operatorname{BiHol}\left(A, \mathbb{C}^{n}\right)$ such that $\psi(A)=A$.

We can consider the action of $\operatorname{Aut}(A)$ on the domain $A$.

Definition 2.2. Given a domain $A \subseteq \mathbb{C}^{n}$ of $\mathbb{C}^{n}$, we say that $\operatorname{Aut}(A)$ is sequences-wise compact when for any point $p \in A$ and for any sequence $\left\{f_{n}\right\}_{n \in \mathbb{N}}$ $\subseteq$ Aut $(A)$, then the sequence $\left\{f_{n}(p)\right\}_{n \in \mathbb{N}} \subseteq A$ does not converge, modulo subsequences, to a boundary point $q \in \partial A$.

We recall that, when $A \subseteq \mathbb{C}^{n}$ is $\phi$-bounded, then $\operatorname{Aut}(A)$ can be endowed with the structure of a Lie group whose topology agrees with the compactopen topology. Moreover, Aut $(A)$ is a compact Lie group if and only if it is sequences-wise compact.

In [4] the explicit description of the group of holomorphic automorphisms of the Kohn Nirenberg domain $D_{K N}$ is given; in particular, Aut $\left(D_{K N}\right)=\mathbb{Z}_{6}$, and so it is compact (as a Lie group). Moreover, in [2] it is proved that the space Aut $\left(D_{K N}\right)$ is sequences-wise compact; but J. Byun could not infer from this result that the group Aut $\left(D_{K N}\right)$ is compact since it is not known whether the Kohn Nirenberg domain is $\phi$-bounded.

According to the previous discussion, the case that the Kohn Nirenberg domain is $\phi$-bounded would perfectly fit in the described picture.

\section{The features of the domain $D$}

Our first remark is that the domain $D$ is bounded.

Lemma 3.1 ( $\phi$-boundedness). The domain $D$ is $\phi$-bounded. More precisely, it is Id-bounded.

Proof. We are going to show that a domain $C$ containing $D$ is Id-bounded. Thus, let

$$
C:=\left\{\left.(z, w) \in \mathbb{C}^{2}|-| w\left|+\frac{1}{5}\right| w\right|^{2}+|w z|^{2}-\frac{8}{7}|z|^{8}+10|z|^{10}<0\right\} .
$$

It is clear that $D \subset C$. We claim that there exists a positive number $R$ such that all the points $(z, w) \in \mathbb{C}^{2}$ with $|z|>R$ lie outside $C$. In fact, we consider the defining function of $C$ as a polynomial of degree two in the $|w|$ variable with coefficients depending on the parameter $z$; then its roots are given by

$$
|w|=\frac{1 \pm \sqrt{1-4\left(\frac{1}{5}+|z|^{2}\right)\left(10|z|^{10}-\frac{8}{7}|z|^{8}\right)}}{2\left(\frac{1}{5}+|z|^{2}\right)} .
$$

Since the term under square root can be made negative under the condition $|z|>R$, with $R$ sufficiently large, we conclude that any point $(z, w) \in \mathbb{C}^{2}$ with $|z|>R$ does not belong to $C$, and so we get our claim. Having achieved the claim, we then discuss the case of $|z| \leq R$. It is clear that a sequence of points 
$(z, w) \in C$ with $|z|<R$ and $|w|$ which goes to infinity cannot occur. Thus $C$, and a fortiori $D$, is Id-bounded.

We are going to prove that the domain $D$ is regular. This computation is elementary and we make it explicit for the reader's convenience.

Lemma 3.2 (Smoothness). The domain D is smooth.

Proof. We are going to show that the defining function has no critical points. We suppose that there is a point which satisfies the three conditions

$$
\begin{gathered}
\rho(z, w)=\operatorname{Re} w+\frac{1}{5}|w|^{2}+|w z|^{2}+|z|^{8}+\frac{15}{7}|z|^{2} \operatorname{Re} z^{6}+10|z|^{10}=0 ; \\
\rho_{w}(z, w)=\frac{1}{2}+\frac{1}{5} \bar{w}+\bar{w}|z|^{2}=0 ; \\
\rho_{z}(z, w)=\bar{z}|w|^{2}+4 \bar{z}|z|^{6}+\frac{15}{2} z^{6} \bar{z}+\frac{15}{14} \bar{z}^{7}+50 \bar{z}|z|^{8}=0 .
\end{gathered}
$$

The second equation holds if and only if

$$
w=\frac{-1}{2\left(\frac{1}{5}+|z|^{2}\right)} .
$$

We notice that at a critical point we cannot have $z=0$. In fact, such a point would be $\left(0,-\frac{5}{2}\right)$, and then we would have both $\rho_{w}=\rho_{z}=0$, but $\rho\left(0,-\frac{5}{2}\right)=-\frac{5}{4} \neq 0$. We also notice that the condition $\rho_{z}\left(z, \frac{-1}{2\left(\frac{1}{5}+|z|^{2}\right)}\right)=0$ now implies that $z^{6}$ is a real number, and moreover $z^{6}$ must be negative. So, we can substitute $\left|z^{6}\right|=-z^{6}=-\operatorname{Re} z^{6}$ both inside equation $\rho_{z}\left(z, \frac{-1}{2\left(\frac{1}{5}+|z|^{2}\right)}\right)=0$ and in $\rho\left(z, \frac{-1}{2\left(\frac{1}{5}+|z|^{2}\right)}\right)=0$. Now, the equation $\rho_{z}\left(z, \frac{-1}{2\left(\frac{1}{5}+|z|^{2}\right)}\right)=0$ can be written as

$$
\rho_{z}\left(z, \frac{-1}{2\left(\frac{1}{5}+|z|^{2}\right)}\right)=\bar{z}\left[\frac{1}{4\left(\frac{1}{5}+|z|^{2}\right)^{2}}+\frac{56-105-15}{14}|z|^{6}+50|z|^{8}\right]=0
$$

from which we infer

$$
\frac{-1}{4\left(\frac{1}{5}+|z|^{2}\right)}=|z|^{6}\left(-\frac{64}{14}+50|z|^{2}\right)\left(\frac{1}{5}+|z|^{2}\right) .
$$

Also, using that the second condition implies that $\frac{1}{2} w+\frac{1}{5}|w|^{2}+|w z|^{2}=0$, the equation $\rho\left(z, \frac{-1}{2\left(\frac{1}{5}+|z|^{2}\right)}\right)=0$ can be written as

$$
\rho\left(z, \frac{-1}{2\left(\frac{1}{5}+|z|^{2}\right)}\right)=\frac{-1}{4\left(\frac{1}{5}+|z|^{2}\right)}-\frac{16}{14}|z|^{8}+10|z|^{10}=0 .
$$

Then, putting (3) into (4), we have

$$
|z|^{6}\left[50|z|^{4}+10|z|^{2}-\frac{64}{14}|z|^{2}-\frac{64}{70}-\frac{16}{14}|z|^{2}+10|z|^{4}\right]=0 .
$$

Equation (5) implies, since we already discussed that a critical point cannot allow $z=0$, that

$$
60|z|^{4}+\frac{60}{14}|z|^{2}-\frac{64}{70}=0
$$


Therefore, taking the positive root,

$$
|z|^{2}=\frac{-\frac{60}{14}+\frac{\sqrt{11652}}{7}}{120}
$$

Finally, it is straightforward to check that a point, satisfying both (2) and (6), does not satisfy $\rho=0$. Thus, the domain $D$ is smooth.

Remark 3.3. Consider the domain

$D_{k, a}:=\left\{\left.(z, w) \in \mathbb{C}^{2}|\operatorname{Re} w+a| w\right|^{2}+|z w|^{2}+|z|^{8}+\frac{15}{7}|z|^{2} \operatorname{Re} z^{6}+k|z|^{10}<0\right\}$,

that is a modification of the Kohn Nirenberg domain with parameters. This is still bounded, for $a$ and $k$ positive numbers. Nevertheless, it can be computed that, for any fixed positive $a$, we can find a positive $k$ such that $D_{a k}$ does not have smooth boundary.

Lemma 3.4 (Levi-convexity). All the boundary points of $D$ are Levi-convex and all but the origin are strongly Levi-convex.

Proof. Let us write $w=|w| \exp (i \alpha)$ and $z=|z| \exp (i \theta)$. To compute the opposite of the determinant of the Levi form, we use the formula (3.10), page 61 , in [10], getting

$$
\begin{aligned}
& -\rho_{w \bar{w}}\left|\rho_{z}\right|^{2}-\rho_{z \bar{z}}\left|\rho_{w}\right|^{2}+2 \operatorname{Re}\left(\rho_{w \bar{z}} \rho_{z} \rho_{\bar{w}}\right) \\
(7) \quad= & {\left[-|w|^{2}+(-16-15 \cos (6 \theta))|z|^{6}\right] \cdot\left[\frac{1}{25}|w|^{2}+\frac{1}{5} \cos \alpha|w|+\frac{1}{4}\right] } \\
& +|z|^{8}\left[|w|^{2}\left(-250|z|^{4}+(-500-16-15 \cos (6 \theta))|z|^{2}-42-30 \cos (6 \theta)\right)\right. \\
(8) \quad & \left.+|w|\left(-200 \cos \alpha|z|^{2}-62 \cos \alpha-\frac{15}{14} \cos (\alpha+6 \theta)\right)-\frac{170}{4}\right] \\
(9) \quad & +|z|^{8}\left[-2500|z|^{12}+\left(-\frac{15033}{98}-\frac{1600}{7} \cos (6 \theta)\right)|z|^{8}-20\right] \\
(10) \quad & -\frac{9}{5}|w|^{4}|z|^{2}+|z|^{18}(-900-800 \cos (6 \theta))+|z|^{14}\left(\frac{1}{5}\left(-\frac{7193}{98}-64 \frac{15}{14} \cos (6 \theta)\right)\right) .
\end{aligned}
$$

The term (7) vanishes for $w=-\frac{5}{2}$. With this value of $w$, the other terms vanish only for $z=0$. But the point $\left(0,-\frac{5}{2}\right)$ does not belong to the boundary of $D$. The term (8) is negative unless $z=0$; the same holds for the term (9). In that case, the term (10) vanishes, while the term (7) vanishes if and only if $w=-\frac{5}{2}$ (case already discussed) or $w=0$, that is, at the origin. Thus, the only boundary point where the determinant of the Levi form vanishes is the origin, while all the other boundary points are strongly Levi-convex, as wanted.

Remark 3.5. Again, not all similar modifications of the Kohn Nirenberg domain keep its properties. In fact, it is not hard to compute that the domain

$$
D_{4,1}:=\left\{\left.(z, w) \in \mathbb{C}^{2}|\operatorname{Re} w+4| w\right|^{2}+|z w|^{2}+|z|^{8}+\frac{15}{7}|z|^{2} \operatorname{Re} z^{6}+|z|^{10}<0\right\} .
$$

has smooth boundary, but it is not a Levi-convex domain. 
For the next result, we follow the argument of J. E. Fornaess and B. Stensones [5] which is a variation of the original argument given by Kohn and Nirenberg. There is a basic observation to make. Suppose that a domain $B \subset \mathbb{C}^{n}$ is locally $\mathbb{R}$-convexifiable at a boundary point $p$. Then in the holomorphic co-ordinates under which the boundary looks convex, the real tangent space $T_{p} B$ leaves the domain $B$ on a half-space. A fortiori, a portion around $p$ of the complex tangent space $H_{p} B \subset T_{p} B$ does not intersect the interior of the domain $B$. That is, $H_{p} B$ is a complex hypersurface of $\mathbb{C}^{n}$ which, around $p$ does not hit the domain $B$. This kind of complex hypersurfaces are called support hypersurfaces. Thus, if we show that any complex hypersurface around $p$ does not intersect $B$, this will be invariant under any holomorphic change of coordinates. What we will conclude is that the domain $B$ is not $\mathbb{R}$-convexifiable around $p$.

We are now ready to present our lemma on non-convexifiability.

Lemma 3.6 (Non $\mathbb{R}$-convexifiability at the origin). The domain $D$ admits no holomorphic support curves at the origin.

Proof. Any holomorphic curve around the origin can be parametrized as $z=$ $\psi(w)$ or as $w=\phi(z)$, where $\phi$ and $\psi$ are holomorphic functions that fix the point 0 . We are going to show that both $\rho(\psi(w), w)$ and $\rho(z, \phi(z))$ change sign on any neighborhood of the origin. Let us consider the case $z=\psi(w)$. As our argument is local, we can write $\psi(w)=a w^{l}+o\left(w^{l}\right)$. Then, the leading term in $\rho\left(a w^{l}+o\left(w^{l}\right), w\right)$ is $\operatorname{Re}(w)$, that changes sign on any small neighborhood of the origin. The remaining case is on $\phi(z)=b z^{m}+o\left(z^{m}\right)$. Here, the leading term of $\rho\left(b z^{m}+o\left(z^{m}\right), z\right)$ depends on $m$. If $m<8$, the leading term is $\operatorname{Re}\left(b z^{m}\right)$, which changes sign on any small neighborhood of the origin. If $m>8$, then the leading term is $|z|^{8}+\frac{15}{7}|z|^{2} \operatorname{Re} z^{6}$, which also changes sign in any small neighborhood of the origin.

Finally, if $m=8$, then the leading term is $\operatorname{Re}\left(b z^{8}\right)+|z|^{8}+\frac{15}{7}|z|^{2} \operatorname{Re} z^{6}$. Again, this expression changes sign in any small neighborhood of the origin. Thus, there is no support function at the origin and this implies that the domain $D$ is not $\mathbb{R}$-convexifiable at the origin.

Remark 3.7. Exactly the same argument works for the domain $A_{K N}=\{\operatorname{Re} w+$ $\left.|z|^{8}+|z|^{2} \operatorname{Re} z^{6}<0\right\}$ (which contains in the boundary the real line $\{\operatorname{Re} w=$ $0, z=0\}$ consisting of weakly spherical points, as already discussed in [6]) and for the domain $D_{K N}$. We can look at the picture this way: as $D_{K N}$ is a modification of $A_{K N}$ which adds the property of strong Levi-convexity at any point except the origin, and in turn $D$ is a modification of $D_{K N}$ which adds the property of boundedness.

Proof of Theorem 1.1. As the defining function of $D$ is clearly analytic, Lemmas 3.1, 3.2, 3.4, 3.6 provide the proof of the theorem.

As an application, we are going to prove the compactness of the automorphism group of our example $D$. 
For our domain $D$, we can apply a theorem by Bedford and Pinchuk [1]. Their result states that, if a smoothly bounded domain $B$ of $\mathbb{C}^{2}$ has real analytic boundary, and if the automorphism group of $B$ is non compact, then the domain $B$ is biholomorphic to the domain

$$
E_{m}:=\left\{\left.(z, w) \in \mathbb{C}^{2}|| w\right|^{2}+|z|^{2 m}<1\right\}
$$

for some positive integer $m$. We are now ready to present our application.

Proof of Corollary 1.2. If Aut $(D)$ were non compact, then by [1] it would be biholomorphic to the domain $E_{m}$. But, for any positive integer $m$, the domain $E_{m}$ is strongly Levi-convex, while $D$ is only weakly Levi-convex at one point. Thus Aut $(D)$ must be compact.

Remark 3.8. In view of the result in [4], it is straightforward to notice that

$$
\operatorname{Aut}(D) \subseteq \operatorname{Aut}\left(D_{K N}\right)=\left\{(z, w) \mapsto\left(e^{i k \frac{\pi}{3}}, w\right) \mid k \in 0, \ldots, 6\right\} \text {. }
$$

\section{References}

[1] E. Bedford and S. Pinchuk, Domains in $\mathbb{C}^{2}$ with noncompact automorphism group, Indiana Univ. Math. J. 47 (1998), no. 1, 199-222.

[2] J. Byun, On the automorphism group of the Kohn-Nirenberg domain, J. Math. Anal. Appl. 266 (2002), no. 2, 342-356.

[3] - On the boundary accumulation points for the holomorphic automorphism groups, Michigan Math. J. 51 (2003), no. 2, 379-386.

[4] J. Byun and H. R. Cho, Explicit description for the automorphism group of the KohnNirenberg domain, Math. Z. 263 (2009), no. 2, 295-305.

[5] J. E. Fornaess and B. Stensones, Lectures on Counterexamples in Several Complex Variables, Princeton University Press, 1987.

[6] J. J. Kohn and L. A. Nirenberg, A pseudo-convex domain not admitting a holomorphic support function, Math. Ann. 201 (1973), 265-268.

[7] M. Kolar, Convexifiability and supporting function in $\mathbb{C}^{2}$, Math. Res. Lett. 2 (1995), no. $4,505-513$.

[8] Generalized models and local invariants of Kohn-Nirenberg domains, Math. Z. 259 (2008), no. 2, 277-286.

[9] R. Narasimhan, Several Complex Variables, University of Chicago press, Chicago 1971.

[10] G. D. Sala, A. Saracco, A. Simioniuc, and G. Tomassini, Lectures on Complex Analysis and Analytic Geometry, Edizioni della Normale, 2006.

Dip. Di Matematica e Informatica "U. Dini"

UNIVERSITA DI FIRENZE

Viale Morgagni 67A, Firenze, Italy

E-mail address: simocala@gmail.com 